\title{
Resolução de algoritmos e de problemas de adição e subtração: uma análise de estratégias utilizadas por estudantes com diagnóstico ou prognóstico de Discalculia do Desenvolvimento
}

\section{Resolution of algorithms and addiction and subtraction problems: an analysis of strategies used by students with diagnosis or prognosis of Developmental Dyscalculia}

\section{Resolución de algoritmos y problemas de suma y resta: un análisis de estrategias utilizadas por estudiantes con diagnóstico o pronóstico de Discalculia del Desarrollo}

\author{
José Ricardo Barbosa Cardoso* \\ Isabel Cristina Machado de Lara*
}

\section{Resumo}

Este artigo apresenta parte dos resultados de uma pesquisa de Mestrado desenvolvida com cinco estudantes que apresentam o diagnóstico ou prognóstico de Discalculia do Desenvolvimento. O objetivo é analisar as estratégias utilizadas pelos participantes para resolver problemas convencionais, comparando-as com as utilizadas para resolver algoritmos. Para tanto os participantes realizaram testes padronizados, dos quais foram selecionados, para este estudo os resultados dos algoritmos e problemas de adição e subtração. Para análise dos dados coletados, optou-se pela Análise Textual Discursiva. Evidencia que para resolução de algoritmos as únicas estratégias utilizadas são o cálculo mental e o uso dos dedos. Em relação à resolução de problemas convencionais do tipo padrão, a estratégia mais utilizada foi o uso de algoritmos, e em seguida, novamente o cálculo mental e o uso dos dedos. Mostra que os estudantes, embora possuam diagnóstico ou prognóstico de Discalculia Operacional apresentam um número maior de erros na execução dos algoritmos de subtração. Contudo, o desempenho na resolução de problemas é satisfatório, apontando que possuem compreensão do conceito de adição e subtração, portanto a Discalculia Ideognóstica não está vinculada ao treinamento do algoritmo.

Palavras-chave: algoritmos; Discalculia do Desenvolvimento; resolução de problemas.

Recebido em: 26/08/2019 - Aprovado em: 03/06/2021

http://dx.doi.org/10.5335/rep.v28i1.9813

Mestre em Educação em Ciências e Matemática pela PUCRS. Licenciado em Matemática pelas Faculdades Porto-Alegrenses/FAPA. Atualmente é, professor do Colégio La Salle Niterói. Orcid: https://orcid.org/0000-0002-0457-1519. E-mail: ricardo_mat86@hotmail.com

** Pós-doutorado em Educação em Ciências e Matemática pela PUCRS. Doutora e Mestre em Educação pela Universidade Federal do Rio Grande do Sul (UFRGS). Licenciada em Matemática pela UFRGS, Professora Permanente do Programa de Pós-Graduação em Educação em Ciências e Matemática da PUCRS. Coordenadora do Grupo de Estudos e Pesquisas sobre Discalculia GEPED/PUCRS. Orcid: https://orcid.org/0000-0002-0574-8590. E-mail: isabel.lara@pucrs.br 


\section{Abstract}

This article presents part of the results of a Master's research developed with five students presenting the diagnosis or prognosis of Developmental Dyscalculia. The objective is to analyze the strategies used by participants to solve conventional problems, comparing them with those used to solve algorithm. Standardized tests were used, being only in addition and subtraction operations. For analysis of the collected data we opted for the Discursive Textual Analysis. It shows that for algorithms resolution the only strategies used are mental calculation and the use of fingers. Regarding conventional problem solving of the standard type, the most used strategy was the use of algorithms, and then again the mental calculation and the use of the fingers. It shows that the students, although having diagnosis or prognosis of Operational Dyscalculia present a larger number of errors in the execution of the subtraction algorithms. However, the problem solving performance is satisfactory, pointing out that the understanding of the concept of addition and subtraction, therefore the Ideognostic Dyscalculia, is not linked to the training of the algorithm.

Keywords: algorithms; Developmental Dyscalculia; troubleshooting.

\section{Resumen}

Este artículo presenta parte de los resultados de una investigación de maestría desarrollada con cinco estudiantes que presentan el diagnóstico o pronóstico de Discalculia del Desarrollo. El objetivo es analizar las estrategias utilizadas por los participantes para resolver problemas convencionales, comparándolos con los utilizados para resolver algoritmos. Para esto, los participantes realizaron pruebas estandarizadas, de las cuales se seleccionaron los resultados de los algoritmos y los problemas de suma y resta para este estudio. Para el análisis de los datos recopilados, optamos por el Análisis textual discursivo. Muestra que para la resolución de algoritmos, las únicas estrategias utilizadas son el cálculo mental y el uso de los dedos. En cuanto a la resolución de problemas convencionales de tipo estándar, la estrategia más utilizada fue el uso de algoritmos, y luego nuevamente el cálculo mental y el uso de los dedos. Muestra que los estudiantes, aunque tienen diagnóstico o pronóstico de Discalculia Operativa, presentan un mayor número de errores en la ejecución de los algoritmos de resta. Sin embargo, el rendimiento de resolución de problemas es satisfactorio, señalando que la comprensión del concepto de suma y resta, por lo tanto, la Discalculia Ideognóstica, no está vinculada a la capacitación del algoritmo.

Palabras clave: algoritmos; Discalculia del Desarrollo; solución de problemas.

\section{Introdução}

A sociedade apresenta, historicamente, a Matemática como uma disciplina em que os estudantes apresentam muitas dificuldades e baixo desempenho em diferentes situações de avaliação. Algumas dessas dificuldades são constatadas por meio de avaliações que verificam o rendimento escolar, geralmente organizado pelo Ministério da Educação de cada país. De acordo com os resultados sobre o desempenho de estudantes da Educação Básica de escolas estaduais e privadas do Brasil, o rendimento em Matemática vem decrescendo constantemente. Entre os testes que apontam isso, destacam-se o Programa Internacional de Avaliação de Alunos (PISA) e a Prova Brasil. Em relação à Prova Brasil, em 2015, constatou-se que $80 \%$ dos estudantes do Ensino Fundamental e Ensino Médio ficaram abaixo do esperado (BRASIL, 2016). 
De acordo com o Instituto Nacional de Estudos e Pesquisas Educacionais Anísio Teixeira (Inep), em 2016, no teste do Pisa, o Brasil ficou na 66 $6^{\underline{a}}$ colocação, atingindo uma pontuação de 337 pontos em relação ao parâmetro que é 490, evidenciando que 70,25\% dos estudantes brasileiros estão abaixo do esperado (INEP, 2016). Considerando que a Matemática é uma área de conhecimento que se constitui como um conjunto de conceitos e ferramentas essenciais para a resolução de problemas do cotidiano e de outras áreas, torna-se relevante ficar atendo a esse nível de desempenho dos estudantes.

Entre as justificativas apontadas para os resultados nessas avaliações, destacam-se as defasagens na aprendizagem devido às dificuldades encontradas pelos estudantes, desde os anos iniciais no Ensino Fundamental, e a formação dos docentes que ensinam Matemática. Em relação a essa formação, evidencia-se, principalmente, a incapacidade do professor de identificar e compreender as dificuldades de aprendizagem manifestadas pelos estudantes.

Dentre essas dificuldades de aprendizagem, verificam-se aquelas de caráter extrínseco ao estudante e aquelas advindas de fatores internos ocasionadas, portanto, por Transtornos de Aprendizagem. Uma definição para Transtornos de Aprendizagem é dada por Ciasca (2003, p, 32), ao afirmar que:

[...] o termo utilizado para indicar uma perturbação ou falha na aquisição e uso de informações, ou na habilidade de solucionar problemas, manifesta-se por dificuldades significativas na aquisição e uso da escrita, fala, leitura, raciocínio e/ou habilidades aritméticas, devido a disfunção do sistema nervoso central, substancialmente abaixo do esperado para a idade, escolarização e nível de inteligência.

É necessário fazer uma distinção entre dificuldade e transtorno de aprendizagem. Dificuldades dizem respeito a falhas no processo de ensino ou de aprendizagem, metodologias inadequadas utilizadas pelos docentes, fatores familiares e psicológicos dos estudantes, bem como aspectos socioeconômicos (RELVAS, 2011). Já, os transtornos ou distúrbios estão relacionados a uma falha no processamento do sistema nervoso central que acarretam em problemas de leitura, escrita e habilidades matemáticas (RELVAS, 2011).

No caso da Matemática, dois transtornos são reconhecidos, a Acalculia e a Discalculia. Neste artigo, são aprofundadas as concepções acerca da Discalculia do Desenvolvimento, uma vez que os estudantes participantes da pesquisa possuem diagnóstico e prognóstico do transtorno. Entre as definições para Discalculia do Desenvolvimento encontradas na literatura, destaca-se a de Kosc (1974, p. 166): 
[...] a structural disorder of mathematical abilities in which it had its origins in genetic or congenital disorders of the parts of the brain which are the physiological anatomo substrate of the maturation of age-appropriate mathematical abilities without a simultaneous disorder of general mental functions. ${ }^{1}$

A Discalculia do Desenvolvimento é um transtorno que acomete entre 3\% e 6\% da polução dos estudantes em idade escolar, ponde variar seu grau de severidade e ter comorbidade com a Dislexia. Trata-se, portanto, de uma amostra considerada rara nas escolas, uma vez que as etapas de diagnóstico não são acessíveis a todos. Diante disso, neste estudo participaram cinco estudantes dos quais um possui laudo de Discalculia, estudante do Ensino Médio e quatro com prognóstico desse transtorno, sendo três estudantes que cursam o Ensino Fundamental e um o Ensino Superior. O objetivo desta pesquisa é analisar as estratégias utilizadas pelos participantes para resolver problemas convencionais e não convencionais, comparando-as com as utilizadas para resolver algoritmo. Para coleta de dados foram utilizados três instrumentos de avaliação: Prova de Aritmética (SEABRA; MONTIEL; CAPOVILLA 2013); Teste de Desempenho Escolar (STEIN, 1994).

\section{Discalculia do Desenvolvimento}

A palavra Discalculia, etimologicamente, deriva dos termos “dis" (desvio) acrescido de "calculare" (calcular, contar). Cohn (1968, p. 202) define o transtorno como "[...] a dificuldade em realizar operações matemáticas, normalmente associadas a problemas de revisualização de números, ideação, cálculo e aplicação de instruções matemáticas".

No Manual Diagnóstico e Estatístico de Transtornos Mentais - DSM-5 (2014, p. 325), o termo Discalculia é nomeado como "Transtorno da Matemática" e sua definição é de:

[...] um termo alternativo usado em referência a um padrão de dificuldades caracterizado por problemas no processamento de informações numéricas, aprendizagem de fatos aritméticos e realização de cálculos precisos ou fluentes. Se o termo discalculia for usado para especificar esse padrão particular de dificuldades matemáticas, é importante também especificar quaisquer dificuldades adicionais que estejam presentes, tais como dificuldades no raciocínio matemático ou na precisão na leitura de palavras.

Esse manual apresenta uma nota de nomenclatura, cuja denominação é em prejuízos na matemática, apontando quatro domínios que podem ser características do distúrbio: senso numérico; memorização de fatos numéricos; precisão ou fluência de cálculos; precisão no raciocínio matemático (DSM-5, 2014). O documen- 
to enfatiza que os principais sintomas são: dificuldades na construção do número que se caracterizam por inversões; na leitura de números; em realizar adições simples; falhas na distinção dos sinais das operações; em decifrar de modo correto o valor dos numerais com vários dígitos; na memorização de dados numéricos; em armar a conta matemática e disposição no espaçamento errado de números com operações de multiplicação e divisão (DSM-5, 2014).

De modo semelhante, a Classificação Estatística Internacional de Doenças e Problemas Relacionados à Saúde - CID-10, refere-se à Discalculia do Desenvolvimento como "Transtorno Específico da Habilidade em Aritmética", com a seguinte definição "[...] uma alteração na capacidade para a realização de operações matemáticas abaixo do esperado para a idade cronológica, nível cognitivo e escolaridade, sem presença de alterações neurológicas ou deficiências sensoriais e motoras" (1993, p. 5). O documento ressalta que os protagonistas do processo são indivíduos que possuem problemas relacionados à parte neurológica e de rendimento escolar inferior aos demais, portanto suas defasagens não são relacionadas a dificuldades físicas e emocionais, mas diretamente percebidas em problemas com cálculos aritméticos e raciocínio matemático (CID-10, 1993). Além disso, corrobora os quatro domínios em defasagem apontados pelo DSM-5 (2014).

Adicionado a isso, vale ressaltar que, segundo Bastos (2016), os estudantes que possuem Discalculia do Desenvolvimento podem ter indícios nos anos iniciais do Ensino Fundamental, mas somente entre as idades de 7 a 8 anos, com a introdução dos símbolos e das primeiras operações básicas que os sintomas podem ficar nítidos. Dessa forma, perceber os sintomas na fase de criança até a juventude é parâmetro para diagnosticar cedo a Discalculia (BASTOS, 2016).

Para dar conta dos objetivos propostos neste estudo, toma-se como alicerce teórico principal os estudos de Kosc (1974), que, além de propor uma definição de Discalculia do Desenvolvimento, propõe categorias que vão além dos quatro domínios previstos pelos manuais, possibilitando uma visão mais detalhada das habilidades que podem estar em defasagem em estudantes com diagnóstico e prognóstico desse transtorno. Kosc (1974) define seis categorias para a Discalculia do Desenvolvimento:

- Discalculia Verbal: indivíduos que apresentam defasagens em quantificar e mencionar numerais, termos e simbologias;

- Discalculia Practognóstica: indivíduos que apresentam defasagens em fazer enumerações, comparações e manipulações de objetos;

- Discalculia Léxica: indivíduos que apresentam defasagens na leitura de simbologias matemáticas; 
- Discalculia Gráfica: indivíduos que apresentam defasagens em realizar a caligrafia dos numerais;

- Discalculia Ideognóstica: indivíduos que apresentam defasagens em realizar cálculos de cabeça e no entendimento de conhecimentos matemáticos;

- Discalculia Operacional: indivíduos que apresentam defasagens em realizar cálculos a partir da utilização da escrita em simbologias matemáticas.

Ressalta-se que neste estudo o foco principal é na Discalculia Operacional e na Discalculia Ideognóstica.

\section{Resolução de algoritmos e de problemas}

Ifrah (1994, p. 95) afirma que “[...] os algoritmos são instrumentos desenvolvidos para tornar o cálculo mais simples por economizar tempo e facilitar sua realização através da generalização dos passos". Complementando essa ideia, pode-se citar Usiskin (1998, p. 7) ao definir algoritmo como um "[...] procedimento ou uma sequência de procedimentos, com um número finito de passos, destinado a executar uma tarefa que se deseja realizar".

De acordo com Toledo e Toledo (1997), cada algoritmo é composto por elementos diferentes, como, por exemplo, tem-se na adição os termos parcela, parcela e total ou soma; na subtração minuendo, subtraendo e resto ou diferença; na multiplicação temos o fator, fator e produto e na divisão dividendo, divisor, quociente e resto. Na prática, percebe-se que nas aulas os estudantes têm a tendência de memorizar os procedimentos do algoritmo para a resolução dos problemas propostos, mas não conseguem ter o discernimento de escolher o caminho para encontrar a resposta.

Contudo, essa memorização não é suficiente para que esse estudante seja considerado numeralizado. De acordo com Nunes e Bryant (1997), identifica-se que um indivíduo está numeralizado quando ele é capaz de utilizar seu raciocínio matemático de maneira significativa e adequada em certas circunstâncias e domina o sistema numérico e as operações aritméticas e pensa com o conhecimento matemático. "É ser capaz de pensar sobre e discutir relações numéricas e espaciais utilizando as convenções [...] da nossa própria cultura" (NUNES; BRYANT, 1997, p. 19). Assim, o mesmo deverá realizar a leitura de enunciados de uma situaçãoproblema e escolher qual operação é apropriada para encontrar sua solução, além disso, deverá identificar as distintas operações matemáticas.

Mesmo diante dessa definição de numeralização, o ensino da Matemática nos anos inicias do ensino Fundamental, ainda tem como objetivo principal para al- 
guns professores a habilidade de resolver algoritmos. Entretanto, de acordo com Kamii e Housman (2002, p. 100), o uso dos algoritmos é considerado prejudicial na aprendizagem por dois fatores: "[...] i) eles encorajam a criança a abandonar seu próprio pensamento; ii) eles "desensinam" valor posicional, desse modo impedindo as crianças de desenvolver o senso numérico”. Nesse sentido, Carraher, Carraher e Schliemann (1988) afirmam que a escola deveria incentivar os estudantes a desenvolverem seu pensamento utilizando um simbolismo que possibilite a comunicação e a troca de experiências. Os autores afirmam ainda que o método de resolução de problemas não elimina a aprendizagem dos algoritmos.

Em suas pesquisas, desenvolvidas com cinquenta estudantes do $1 \square$ ao $5 \square$ ano do Ensino Fundamental, Lara (2011, p. 17) mostra que:

[...] a partir do momento em que as crianças começavam a lidar com algoritmos pareciam iniciar um processo de desligamento do seu próprio modo de pensar, afastando-se cada vez mais de um pensamento flexível e da capacidade de fazer estimativas e interpretações.

Lara (2011, p. 119) comprova que "[...] na medida em que eles avançam na vida escolar, tendem a abstrair o seu pensamento de modo a optar cada vez mais pelo uso dos algoritmos". Contudo, isso pode implicar no abando do seu próprio modo de pensar bem como na desistência da busca de estratégias próprias para resolver problemas (LARA, 2011).

No que concerne à resolução de problemas, D’Amore (2007, p. 285) argumenta que: “[...] é a forma mais eficaz não somente do desenvolvimento da atividade matemática dos estudantes, mas também da aprendizagem dos conhecimentos, das habilidades, dos métodos e de suas aplicações”. Contudo, faz necessário repensar a concepção de problema. Villa e Callejo (2006, p. 29) ressaltam que:

Um problema é uma situação, proposta com finalidade educativa, que propõe uma questão matemática, cujo método de solução não é imediatamente acessível ao aluno ou ao grupo de alunos que tenta resolvê-la, porque não dispõe de um algoritmo que relaciona os dados e a incógnita ou de um processo que identifique automaticamente os dados com a conclusão e, portanto, deverá buscar, investigar, estabelecer relações e envolver suas emoções para enfrentar uma situação nova.

Diante disso, um problema não seria resolvido facilmente com uma simples aplicação de algoritmo. Nesse sentido, Brito (2006) aborda o termo "solução" para comentar sobre as estratégias de se chegar a uma resposta de um problema. Em relação ao termo de solução de problemas, Brito (2006, p. 27) entende que “[...] é uma forma complexa de combinação dos mecanismos cognitivos disponibilizados a partir do momento em que o sujeito se depara com uma situação para a qual precisa bus- 
car alternativas de solução". Portanto, a solução de problemas remete a um processo que começa quando o indivíduo encontra uma circunstância que provoca a vontade pela busca de uma solução e na reorganização dos elementos que constam na estrutura, de uma maneira que chegue a um resultado (BRITO, 2006). Em relação às classificações sobre os problemas matemáticos, Diniz (2001) denomina-os como convencionais e não convencionais. Assim, segundo a autora (2001, p. 100-101),

[...] as características básicas de um problema convencional são: texto na forma de frases, diagramas ou parágrafos curtos; os problemas vêm sempre após a apresentação de determinado conteúdo; todos os dados de que o resolvedor necessita aparecem explicitamente no texto e, em geral, na ordem em que devem ser utilizados nos cálculos; os problemas podem ser resolvidos pela aplicação direta de um ou mais algoritmos; a tarefa básica em sua resolução é identificar que operações são apropriadas para mostrar a solução e transformar as informações do problema em linguagem matemática; a solução numericamente correta é um ponto fundamental, sempre existe e é única.

De acordo com Brito (2006), o uso de problemas convencionais pode gerar alguns comportamentos inoportunos nos estudantes quando estão realizando a resolução, pois é comum os discentes vincularem esses problemas com alguns cálculos aritméticos e indagarem: "Qual a conta que tenho que fazer nesse problema?", ou tentarem identificar nos problemas termos que associam a linguagem à alguma operação, como: "ao todo", "total" ou "acrescentar" que podem ser direcionados a natureza das operações de adição e "restou", "sobrou", "faltou" e "perdeu" que estão relacionados ao campo da subtração. Isso leva a ter um desequilíbrio na aprendizagem se tratando da resolução de problemas, pois os estudantes tendem a pensar só no algoritmo que deve escolher sem pensar nos conceitos envolvidos no problema, buscando memorizar como resolve cada tipo de problema. Efeito disso pode ser um fracasso na aprendizagem se apegando cada vez mais ao uso de um algoritmo e deixado de lado seu próprio raciocínio, como mostra Lara (2011) em seus estudos. Esse tipo de comportamento pode causar o medo, insegurança e ao longo de sua vida escolar ter defasagens que dificultam sua aprendizagem matemática (DINIZ, 2011).

A resolução de problemas, conforme Polya (2006), é um processo cujo objetivo é a investigação de um ato para atingir um propósito que deseja alcançar, mas que não se pode ter certeza se vai conseguir concretizá-lo, dessa forma estabeleceu quatro etapas para a solução de problemas: 1) Compreender o problema: por meio da leitura o estudante precisa identificar palavras, significados e expressões com o objetivo de descobrir a solução; 2) Elaboração de um plano: após a leitura deve organizar os processos e estratégias que julga adequados para encontrar a solução; 3) Executar o plano: deverá escolher pela melhor estratégia e executá-la; 4) Re- 
trospecto: nessa última etapa o estudante deverá apurar e reconhecer a resposta encontrada a partir da circunstância inicial do problema.

Na mesma perspectiva de Polya, Dante (1991) renomeia algumas etapas: 1) compreender o problema; 2) elaboração do plano; 3) execução; 4) retrospecto ou verificação. De acordo com Dante (1991), as etapas estabelecidas auxiliam os estudantes na organização do seu pensamento na resolução de problemas. Percebe-se que, ao longo do tempo, as etapas para a resolução de problemas foram sendo aprimoradas por cada autor com intuito de auxiliar os estudantes a resolverem os problemas propostos.

\section{0 campo conceitual da estrutura aditiva}

Com base em alguns conceitos da teoria de Piaget, em particular de esquema e dos estudos de Vygotsky sobre linguagem, Gérard Vergnaud propôs a Teoria dos Campos Conceituais (LARA, 2011). De acordo com Golbert (2002, p. 28), Vergnaud define que um campo conceitual é "[...] um conjunto de situações, cujo tratamento implica esquemas, conceitos e teoremas, em estreita conexão, assim como as representações linguísticas e simbólicas, suscetíveis de serem utilizadas para representá-lo”. A autora complementa que, para Vergnaud, existem três aspectos para os campos conceituais: situações; conceitos; e, representações simbólicas.

Relacionando a aprendizagem a esses três aspectos, percebe-se que um indivíduo necessita lidar com diversas situações, seja pela aprendizagem escolar, seja por suas vivências fora do ambiente escolar. Tais situações lhe propiciarão a criar esquemas para saber enfrentá-las, e assim irá se apoderar de representações simbólicas que se agregará às situações para saber solucioná-los. Será a partir do movimento desses três aspectos que o indivíduo estará criando conceitos, de acordo com a teoria de Vergnaud (GROSSI, 2001).

O campo conceitual das estruturas aditivas é uma união de circunstâncias, cujo princípio acarreta em uma ou inúmeras adições ou subtrações, ou combinação das duas, e um agrupamento de conceitos e teoremas que possibilitam averiguar as circunstâncias como afazeres matemáticos (VERGNAUD, 1996). Conforme Kamii (1998, p. 84), com perspectivas piagetianas, a operação de adição possui como princípio “[...] uma ação mental (abstração construtiva) de combinar dois totais para criar um de ordem superior no qual os totais anteriores, se tornam duas partes". Já a subtração, é considerada pela autora como mais complicada do que a adição, devido aos seus níveis de entendimento. Assim, na adição o estudante tem como 
princípio "ascender" de dois totais (6 e 3, por exemplo) para uma totalidade superior (9), enquanto na subtração, $(9-6)$, ele precisa descender do total (9) para parte (6) e depois ascender da parte (3) para encontrar o total (9) (KAMII,1998).

\section{Participantes da pesquisa e instrumentos de coleta de dados}

Buscaram-se estudantes que possuíam laudos ou prognósticos de transtornos de aprendizagem em Matemática. Para referir-se a cada um deles, durante a pesquisa, criaram-se codinomes, expostos no Quadro 1, juntamente com algumas informações básicas.

Quadro 1 - Descrição básica dos participantes da pesquisa

\begin{tabular}{|c|c|c|c|c|c|}
\hline Codinome & Gênero & Idade & $\begin{array}{l}\text { Ano em que } \\
\text { estuda }^{*}\end{array}$ & $\begin{array}{c}\text { Modalidade de } \\
\text { escola }\end{array}$ & Município \\
\hline Antônio & Masculino & 15 & $9^{\circ}$ ano $\mathrm{EF}$ & Privada & Porto Alegre \\
\hline Taíssa & Feminino & 17 & 3 ano EM & Privada & Canoas \\
\hline Paulo & Masculino & 15 & $7^{\circ}$ ano EF & Pública & Porto Alegre \\
\hline Ricardo & Masculino & 10 & $5^{\circ}$ ano EF & Pública & Caçapava do Sul \\
\hline Carlos & Masculino & 19 & $4^{\circ}$ sem ES & Privado & Porto Alegre \\
\hline
\end{tabular}

Fonte: elaboração dos autores.

* EF - Ensino Fundamental; EM - Ensino Médio; ES - Ensino Superior.

Além disso, coletaram-se todas as informações que os responsáveis possuíam sobre anteriores avaliações e possíveis diagnósticos. Para apresentá-las, organizou-se uma síntese sobre cada participante. Vale ressaltar, que nem toda a documentação foi disponibilizada pelos responsáveis ou pelas escolas. Desse modo, não foi possível obter maiores informações sobre os mesmos aspectos para cada participante. Em todo momento do desenvolvimento da pesquisa, foram mantidas em anonimato as identidades dos participantes.

Para a coleta de dados, foram realizados os seguintes testes: Subteste de Aritmética (STEIN, 2004) e Prova de Aritmética (SEABRA; MONTIEL; CAPOVILLA, 2013).

\section{Analisando 0 desempenho na resolução de algoritmos}

No Quadro 2, foram organizadas as respostas dos Subteste de Aritmética de Stein (1994) e de Seabra, Montiel e Capovilla (2013), contendo os números de acertos (A) e o número de erros (E). 


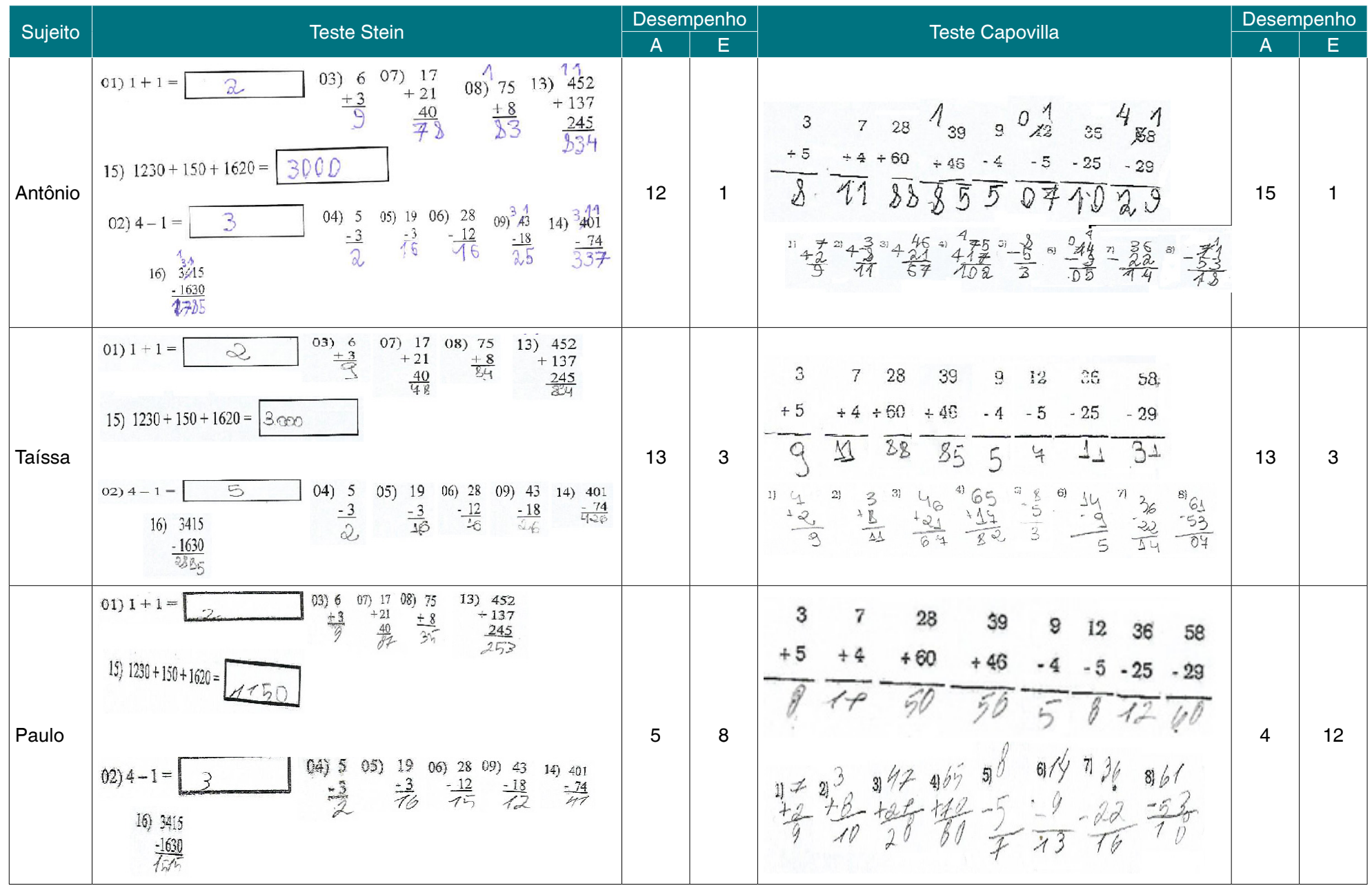


(conclusão)

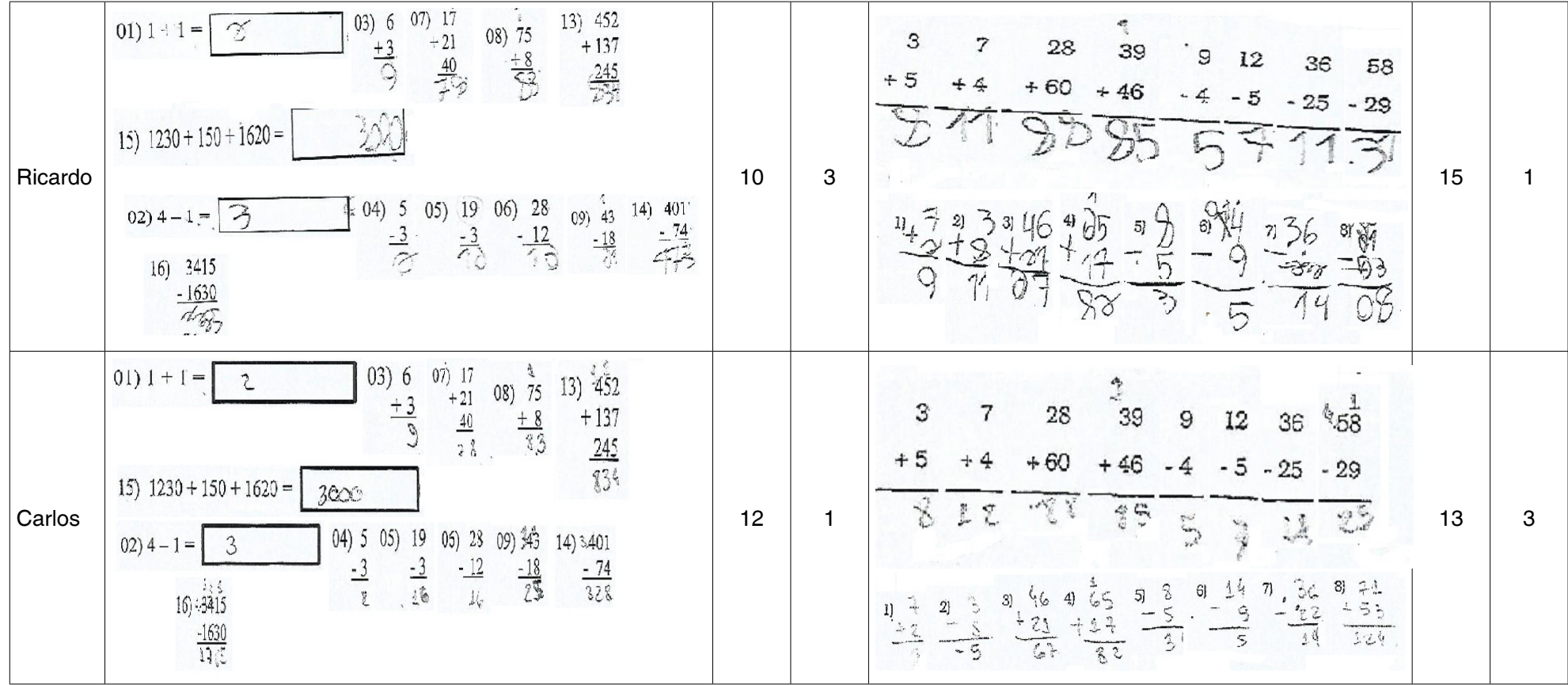

Fonte: elaboração dos autores. 
O primeiro sujeito a realizar os testes foi Antônio, que obteve dois erros com operações da adição e subtração proposto em cada atividade. O primeiro erro foi a partir de uma subtração (401-74) no Subteste de Aritmética (STEIN, 1994) encontrando como resposta o resultado 337. No Teste de Aritmética (SEABRA; MONTIEL; CAPOVILLA, 2013), ao ouvir a operação $(65+17)$ o mesmo registrou (75+17) encontrando como resposta 102, cálculo que deveria ter como resposta 92. A estratégia utilizada por esse estudante foi cálculo mental. Verifica-se que o estudante já havia acertado outras subtrações com retorno e adições com transporte, o que sugere que esses erros ocorreram devido à desatenção em resolver as operações. Sobre isso, Consenza e Guerra (2011) afirmam que existem algumas áreas que auxiliam na aprendizagem como: emoção; memória; atenção; motivação; socialização. No caso da atenção, Brandão (1991) afirma que é o caminho da mente e o agrupamento de procedimentos psicológicos que proporcionam o foco em certo acontecimento ou objeto, com intuito de que o indivíduo possa fazer escolhas, selecionar e planejar os dados que acha significativo. $O$ momento de avaliação sempre pode causar um pouco de ansiedade e angústia para o estudante fazendo com que seu foco de atenção seja outro que não a tarefa que está sendo solicitada naquele instante.

A estudante Taíssa obteve alguns erros em ambos os testes envolvendo algoritmos da adição e subtração. Os erros em ambos os testes foram variados, embora na adição prevaleçam os erros quando necessita de transporte, foi o caso de $(75+8)$ e de $(452+137+245)$, o erro em $(3+9)$ contradiz um padrão ou a não construção do sistema das dezenas. Já na subtração, todos os erros foram cometidos nos algoritmos em que era necessário o retorno de uma ordem para uma ordem inferior, $(43-18=26),(401-74=427),(3415-1630=2885),(58-29=31)$ e $(61-53=7)$, porém não existe um padrão no tipo de erro. Isso pode corroborar a não construção dos sistemas tanto de dezena quanto da centena, apontando para um erro de processamento numérico, o que poderá ocasionar dificuldades no cálculo. Seu desempenho vai ao encontro do que consta em seu parecer psicológico e psicopedagógico os quais apontam déficits em habilidades visoespaciais e visoperceptivas que causam dificuldades em realizar operações aritméticas, tendo como diagnóstico final pelo CID 10, o transtorno F81.2, ou seja, Discalculia do Desenvolvimento. De fato, os erros cometidos por Taíssa são característicos da Discalculia, uma vez que entre as defasagens apresentadas nesse transtorno, conforme Bastos (2016), destaca-se a habilidade para realizar cálculos simples, memorização de fatos numéricos e dificuldades em resolver operações com transporte. Além disso, em relação à catego- 
rização realizada por Kosc (1974), Taíssa apresenta indícios fortes de Discalculia Operacional.

O estudante Paulo ao resolver os testes teve alguns acertos nas adições e subtrações de unidade por unidade, exceto no caso de $3+8=10$. Em relação a outros erros, nas adições e subtrações com dois ou mais algarismos o estudante acertou somente uma operação como $(19-3=16)$, demonstrando defasagem no processamento numérico, em particular na construção do número e seu valor posicional, embora que a maioria dos erros não estabelecem um padrão. No Subteste de Aritmética (STEIN, 1994), os erros em relação à adição foram: $(17+21+40=87)$; $(75+8=35) ;(452+137+245=253)$ e $(1230+150+1620=4150)$. Na subtração os erros foram os mesmos cometidos pela adição, ou seja, a não compreensão do sistema de numeração decimal e não existência de um padrão, exemplo disso são casos como: $(28-12=15) ;(43-18=12) ;(401-74=41)$; e, $(3415-1630=1515)$. Ou seja, percebem-se erros tanto nas adições e subtrações simples quanto nas de transporte e retorno, respectivamente.

Para compreender possíveis estratégias utilizadas pelos estudantes para encontrar a resposta, é possível citar Kamii (1998) ao tratar que é necessário a criança realizar operações, escrever e identificar os numerais, assim criando um pensamento mental para a utilização da contagem e leitura dos numerais, não acarretando em uma mera memorização de sequências de números. Contudo, no caso de Paulo, nem a memorização de fatos aritméticos foram observados.

O estudante Ricardo ao realizar o Subteste de Aritmética (STEIN, 1994) não apresentou nenhum erro nas operações de adição, mas apresentou três erros na subtração. Na primeira operação $(43$ - 18) encontrou como resposta o número 21 . Já nas outras duas, $(401-74=473)$ e $(3415-1630=2225)$, é observável que o estudante desenvolveu seu cálculo da subtração retirando sempre do algarismo de maior módulo, não levando em conta as regras da subtração, nem mesmo questões do processamento numérico considerando os valores relativos de cada numeral. $\mathrm{Na}$ Prova de Aritmética (SEABRA; MONTIEL; CAPOVILLA, 2013), Ricardo utilizou a mesma estratégia equivocada, pois na operação $(58$ - 29) encontrou como resposta 31. Já em relação as demais operações que não exigiam realizar subtrações com reserva, ou seja, com retorno, o estudante acertou todos os cálculos propostos.

De acordo com Kamii (1998, p.101): "Quando se ensina a criança regras ou operações que elas não entendem, elas perdem a intuição que tinham e têm de ler todos os dígitos como unidades", talvez isso justifique pensar em 9 - 8 e não o $8-9$ como solicitado. Além disso, Ricardo em suas respostas representou números espe- 
lhados, que conforme argumenta Zorzi (2001), crianças que possuem esse tipo de registro em avalições e atividades tende ao possível quadro de Dislexia, envolvendo dificuldades quanto ao domínio de relações espaciais e temporais relacionadas ao conhecimento da escrita, reforçando erros nas letras e nos números. Embora Ricardo não apresente um laudo oficial de Dislexia, são nítidas as suas dificuldades com a leitura e a com a escrita, fato esse corroborado pela fala de sua mãe.

Para compreender os erros cometidos pelos estudantes, vale sublinhar o que Cury (2007, p. 63) afirma, “[...] ao analisar as respostas dos alunos, o fundamental não é o acerto ou o erro em si, mas as formas de se apropriar de um determinado conhecimento, que pode indicar dificuldades de aprendizagem".

O estudante Carlos apresentou desempenho favorável ao realizar o Subteste de Aritmética (STEIN, 1994), acertando todas as operações de adição e de subtração, exceto a operação $(401-74=328)$, ou seja, encontrando um valor a mais que a resposta certa (327), o que leva a sugerir que foi uma falta de atenção. Na Prova de Aritmética (SEABRA; MONTIEL; CAPOVILLA, 2013), Carlos apresentou um erro na adição $(7+4=12)$ e na parte oral o mesmo apresentou dois erros $(3-8=-5)$, onde a proposta era $(3+5)$ e $(71+53=124)$ onde foi lhe ditado $(71-53)$. Percebe-se que, no segundo erro, talvez Carlos tivesse feito a adição mental e, devido à pressa em fazer o registro, acabou invertendo os numerais, o que caracterizaria uma falta de atenção por parte do estudante, embora, por outro lado, considerando números inteiros como seu universo, o estudante acertou o cálculo que se propôs a realizar. O mesmo acontece quando lhe é ditada uma adição e, por distração, realiza uma subtração. Verificando a avaliação psicopedagógica que foi disponibilizada pelo estudante, percebe-se um avanço em seu desempenho, pois percebe-se que não é mais tão lento, como consta no laudo fornecido em 2016, e em geral não apresenta dificuldades em realizar operações de adição, apenas alguns erros que não seguiram um padrão, sugerindo, portanto, uma desatenção.

Em relação aos erros, Fiorentini (2006, p. 4) afirma que:

[...] erro escolar, na verdade, resulta do esforço dos alunos em participar do processo de aprendizagem, produzindo e negociando, a partir de seu mundo e de sua cultura, sentidos e significados sobre que se ensina e aprende na escola. E nesse sentido, o erro não poderia ser visto como um mal a ser erradicado, mas como parte do processo de aprender e desenvolver-se intelectualmente.

É perceptível que os participantes da pesquisa cometeram muito mais erros nos algoritmos de subtração do que nos de adição, corroborando o que Kamii (1998) afirma em relação à subtração ser uma operação que envolve esquemas mais com- 
plexos que a adição, principalmente, frente à noção de negatividade, que muitas vezes não é desenvolvida pelo professor desde os anos iniciais. Durante a realização das atividades propostas, os sujeitos relataram que a subtração é mais difícil que a adição, pois tinham que lembrar como realizavam os seus procedimentos, em particular o "pegar emprestado". O fato de alguns afirmarem que não lembravam de como "pedir emprestado" já demonstra uma não construção do valor posicional e a compreensão de que uma unidade maior vale dez vezes a ordem anterior. De acordo com Ramos (2009), na operação de subtração não acontece o procedimento de "emprestar", mas o processo de desmanchar grupos quando é necessário, fazer trocas por meio de uma estrutura lógica do sistema de numeração decimal, integrando e reintegrando quantias de dez em dez. Trata-se, conforme outros autores, do domínio do processamento numérico. O que conforme as características descritas no DSM-5 (2014), poderiam se referir a prejuízos na memorização de fatos aritméticos, precisão ou fluência de cálculo. Isto é, dois dos quatro domínios listadas pelo manual como aqueles que podem apontar para o Transtorno Específico da Aprendizagem em Matemática, para Discalculia do Desenvolvimento. Contudo, entre os participantes aquele que mais se aproximaria de um prognóstico desse transtorno seria Paulo.

Ao final da análise das estratégias utilizadas, preponderou na resolução de algoritmos, o cálculo direto do algoritmo, sem a tentativa de uma alternativa diferenciada, apenas o cálculo mental, e a contagem nos dedos utilizado por alguns estudantes para pequenas quantidades. Isso pode ser um sinal de que em sala de aula eles já tenham desenvolvido a habilidade de resolver algoritmos de forma mnemônica.

Para os estudantes que não possuem laudo, mas apresentaram, durante a resolução dos algoritmos fortes indícios de uma Discalculia Operacional, é fundamental uma análise mais acertada de uma equipe multidisciplinar e a realização de neuroimagens. Conforme Consenza e Guerra (2011), por meio de neuroimagem é possível visualizar uma ativação do lobo parietal quando os sujeitos estão realizando comparações de quantidades. Dessa forma, uma lesão nessa região pode acarretar problemas em realizar operações aritméticas, mas não perdem a noção de realizar os cálculos por estimativa por meio do hemisfério direito.

\section{Analisando o desempenho na resolução de problemas}

Esta seção tem o objetivo de apresentar as estratégias dos participantes de pesquisa acerca da resolução de problemas convencionais envolvendo o algoritmo 
da adição e subtração. Por meio dos aportes teóricos utilizados, nesta pesquisa, sobre resolução de problemas, elencaram-se cinco categorias a priori, que por meio de uma ATD, com base em Moraes e Galiazzi (2011), serviram para analisar as estratégias utilizadas pelos estudantes participantes da pesquisa, sejam elas: representação pictórica; cálculo mental; uso de algoritmo; contagem nos dedos; oralidade.

Para avaliar as estratégias criadas por cada um dos sujeitos na resolução de problemas convencionais, a partir das categorias a priori, utilizaram-se as seguintes situações-problema:

Situação 1: João tinha quatro maçãs e ganhou mais oito. Com quantas maças João ficou? (SEABRA; MONTIEL; CAPOVILLA, 2013)

Situação 2: Maria tinha treze livros mas perdeu dois. Com quantos livros Maria ficou? (SEABRA, MONTIEL; CAPOVILLA, 2013)

Para organizar os dados da resolução dos problemas a partir do registro dos sujeitos de pesquisa, elaboraram-se quadros com as respostas dos estudantes.

Situação 1: Para resolver a situação: João tinha quatro maçãs e ganhou mais oito. Com quantas maças João ficou? Os sujeitos utilizaram diferentes estratégias, organizadas no Quadro 3.

Quadro 3 - Estratégias utilizadas para resolver a situação problema 1

\begin{tabular}{|c|c|c|c|c|c|c|}
\hline Estudante & Antônio & Taíssa & Paulo & Ricardo & Carlos \\
\hline Resolução & $\frac{44}{12}$ & $\frac{4}{8}$ & & & \\
\hline Estratégia & Algoritmo & Contagem nos dedos & Cálculo mental & Algoritmo & Cálculo mental \\
\hline
\end{tabular}

Fonte: elaboração dos autores.

É perceptível que as estratégias utilizadas pelos estudantes foram distintas, abrangendo três das categorias a priori propostas. $\mathrm{O}$ problema proposto para os estudantes é classificado de acordo Golbert (2002, p.48) como "Composição - dois estados fixos se unem num terceiro estado fixo". Em relação à leitura dos problemas, Antônio, Taíssa e Carlos não necessitaram da ajuda do aplicador, já Ricardo e Paulo solicitaram a ajuda da leitura do problema por possuírem o prognóstico de Dislexia. Para Ricardo, devido a sua dificuldade, foi necessário ler duas vezes o pro- 
blema para que ele abstraísse os dados. Mesmo assim foi solicitado que o estudante lesse o problema em uma leitura mais rápida para o entendimento do enunciado.

Antônio, Paulo, Ricardo e Carlos conseguiram chegar à resposta correta, exceto Taíssa que encontrou a resposta incorreta do problema. A partir disso, a estudante foi indagada de como resolveu o problema e a mesma relatou que "o problema dizia que tinha quatro maçãs e ele ganhou mais quatro maçãs, por isso que encontrei como resposta o número 8". A partir do erro cometido por Taíssa, sugere-se que foi apenas falta de atenção, mas vale acrescentar o dito por Cândido (2001) ao enfatizar que é necessário trabalhar com textos nas aulas de Matemática, pois os estudantes precisam se apropriar da linguagem matemática e dos símbolos utilizados nos conceitos desenvolvidos, assim possibilitando uma compreensão das articulações e expressões do conhecimento construído.

Situação 2: Para resolver a situação: Maria tinha treze livros, mas perdeu dois. Com quantos livros Maria ficou?, os participantes utilizaram diferentes estratégias, organizadas no Quadro 4.

Quadro 4 - Estratégias utilizadas para resolver a situação problema 2

\begin{tabular}{|c|c|c|c|c|c|}
\hline Estudante & Antônio & Taíssa & Paulo & Ricardo & Carlos \\
\hline \multirow{2}{*}{ Resolução } & -13 & 13 & & & \\
& $\frac{1}{11}$ & $\frac{1}{11}$ & & & \\
\hline Estratégia & Algoritmo & Algoritmo & Contagem nos dedos & Algoritmo & Cálculo mental \\
\hline
\end{tabular}

Fonte: elaboração dos autores.

Nessa situação-problema, todos os participantes conseguiram chegar à resposta correta. Os sujeitos Paulo e Ricardo necessitaram que o aplicador fizesse a leitura do problema para realizar a atividade. Para resolver essa situação a maioria dos estudantes optou pela realização do algoritmo e apenas um fez o cálculo mentalmente.

Embora a magnitude dos números envolvidos em ambas situações-problema seja bem menor dos envolvidos na resolução de algoritmos, é perceptível que os estudantes entendem a adição como um esquema de acrescentar e a subtração como um esquema de retirar. Percebe-se que pensam matematicamente para encontrar essas soluções, demonstrando defasagens voltadas em particular à Discalculia 
Operacional e não ao entendimento de conhecimentos matemáticos característicos à Discalculia Ideognóstica.

\section{Confluências e divergências}

Ao referir-se aos resultados encontrados e à análise das estratégias desenvolvidas pelos cinco participantes em resolver algoritmos da adição e subtração e para resolver problemas convencionais, utilizando como instrumentos testes padronizados, é possível perceber algumas confluências. Além disso, baseando-se no desempenho dos cinco estudantes em resolver algoritmos de adição e subtração e problemas convencionais, nota-se que as estratégias utilizadas em cada processo foram distintas.

Vale ressaltar que Antônio, Taíssa, Ricardo e Carlos nas tarefas que envolviam resolução de algoritmos não cometeram muitos erros. Analisando o desempenho de Antônio e Carlos, verifica-se que ambos demonstraram traços de desatenção, uma vez que cometeram erros aleatórios que não demonstraram um padrão. Já Taíssa e Ricardo evidenciaram erros acerca do processamento numérico explicitados durante a resolução de adições com transporte e subtrações com retorno. Em relação a Paulo, percebem-se erros mais básicos, o que sugere a não construção do número e do valor relativo e absoluto dos numerais, o que condiz com sua capacidade de processamento numérico.

Em relação aos problemas convencionais, os participantes tiveram um ótimo desempenho. Nas dez soluções apresentadas, verificou-se que a estratégia mais utilizada foi a montagem do algoritmo, apenas três soluções foram encontradas por meio do cálculo mental e duas pela contagem dos dedos. $\mathrm{O}$ único erro foi cometido por Taíssa, mas sua argumentação leva a pensar que pela rapidez de sua resposta e processamento mental confundiu uma das partes da adição com o total.

Embora a magnitude das quantidades expressas nas situações-problema seja bem menor que as envolvidas nos algoritmos, o que não possibilitaria detectar os mesmos erros no processamento numérico frente a adições com transporte e subtrações com reservas, o objetivo com a proposição dessas duas situações-problema consistiu na possibilidade de analisar se esses cinco estudantes dominam os conceitos de adição e subtração. Diante disso, foi perceptível que embora todos possam possuir algum tipo de dificuldade diante a resolução de um algoritmo, todos entendem que em uma situação de acréscimo está envolvido o conceito de adição e que em uma situação de retirar está envolvido o conceito de subtração. 
Verificou-se que, durante os momentos de aplicações dos problemas convencionais, a maioria dos participantes não conseguiu e nem tentou expressar de modos distintos sua resolução. Isso, por um lado, conforme o relato de alguns, pode ser ocasionado pelo fato de seus professores não estimularem a utilização de outras estratégias para resolução de problemas, por outro, pode estar relacionado à crença de que um problema matemático possui apenas uma forma de resolução e apenas uma resposta correta, evidenciando, como já feito em outras pesquisas, que na escola alguns professores supervalorizam o uso do algoritmo.

Essa prática dos professores é criticada por Diniz (2001, p. 89) ao afirmar que a resolução de problemas “[...] trata de situações que não possuem solução evidente e que exigem que o resolvedor combine seus conhecimentos e decida pela maneira de usá-los em busca da solução". Corroborando a ideia de Diniz, as autoras Lara e Pimentel (2015) ressaltam que os estudantes ao encontrar problemas padronizados, irão resolver de forma automatizada os seus procedimentos.

É relevante destacar, que este artigo apresenta apenas uma parte da pesquisa, e que em outras tarefas, em que a resolução de problemas não convencionais foi analisada, verificaram-se outras estratégias como a utilização de representação pictórica e oralidade.

\section{Considerações finais}

Este artigo teve como objetivo analisar as estratégias utilizadas pelos participantes para resolver problemas convencionais, comparando-as com as utilizadas para resolver algoritmos. Percebeu-se que, ao resolver as operações, os estudantes apresentaram dificuldade em realizar adições com transporte e subtrações com retorno evidenciando defasagens em relação ao processamento numérico. Contudo, na resolução de problemas que abordavam as mesmas operações, e que tinham o intuito de verificar se os estudantes dominam o conceito de adição e subtração o desempenho foi satisfatório, embora Taíssa tenha errado o problema envolvendo adição, evidenciou-se que cometeu o erro por falta de atenção. Vale ressaltar que um dos participantes envolvidos na pesquisa possui diagnóstico de Discalculia Operacional, três participantes possuem fortes indícios de Discalculia Operacional em comorbidade com a Dislexia e um com fortes indícios de Discalculia Operacional e Ideagnóstica.

As estratégias utilizadas pelos participantes para resolver os algoritmos da adição e subtração foram distintas como: cálculo mental; uso dos dedos. Coinciden- 
temente, as mesmas estratégias foram utilizadas para resolver os problemas propostos que foi convertido em algoritmo pela maioria. Acredita-se que isso ocorreu por tratar-se de problemas convencionais que explicitam em seu enunciado a operação aritmética a ser utilizada. Diante disso, por ser considerado um problema do tipo padrão e, geralmente, o mais abordado em sala de aula, os estudantes acabam automatizando as resoluções por meio do algoritmo adotado. Contudo, o fato de demonstrarem o entendimento dos conceitos de adição e subtração apontam para a possibilidade desses estudantes conseguirem pensar matematicamente utilizando as operações matemáticas de modo adequado. Vale sublinhar, que em um estudo mais aprofundado com estes estudantes, Cardoso (2019) evidencia que quando solicitado que resolvam problemas não-convencionais, sejam eles de lógica, sem solução, com excesso de dados ou com mais de uma solução, muitas vezes buscam por outras estratégias de resolução, principalmente a representação pictórica como apresentado por Lara (2011) em seus estudos.

Isso aponta que, frente a um estudante que possui um prognóstico ou um diagnóstico de Discalculia, aconselha-se que o professor crie condições que possibilitem ao estudante pensar flexivelmente frente à resolução de problemas. $\mathrm{O}$ treinamento de algoritmos não garante que o estudante desenvolverá, neste caso, a sua estrutura aditiva e pense matematicamente para resolver problemas. É necessário que o professor estimule a criação de outras alternativas de resolução e que não abandone seu pensamento flexível. Adicionado a isso, o fato de um estudante ter dificuldades com operações aritméticas, o que caracteriza uma Discalculia Operacional, não significa que ele seja incapaz de resolver problemas em que necessite operacionalizar conceitos por meio de estratégias distintas, dificuldade essa relacionada à Discalculia Ideognóstica.

\section{Nota}

1 “[...] um transtorno estrutural de habilidades matemáticas, na qual teve suas origens em transtornos genéticos ou congênitos das partes do cérebro que são o substrato anátomo fisiológico da maturação das capacidades matemáticas adequadas à idade, sem um transtorno simultâneo de funções mentais gerais" (tradução nossa).

\section{Referências}

BASTOS, J.A. Matemática: distúrbios específicos e dificuldades. In: ROTTA, N. T.; OHLWEILER, L.; RIESGO, R. S. (org.). Transtorno de aprendizagem: abordagem neurobiológica e multidisciplinar. Porto Alegre: Artes Médicas, 2016. p. 56-58. 
BRANDÃO, M. L. As bases psicofisiológicas do comportamento. São Paulo: E.P.U., 1991.

BRASIL. Ministério da Educação. Brasil no PISA 2015: Análises e reflexões sobre o desempenho dos estudantes brasileiros. São Paulo: Fundação Santillana, 2016.

BRITO, M. F. Solução de problemas e a matemática escolar. Campinas: Alínea, 2006.

CÂNDIDO, P. Comunicação em Matemática. In: SMOLE, K. S.; DINIZ, M. I. (org.). Ler, escrever e resolver problemas: habilidades básicas para aprender matemática. Porto Alegre: Artes Médicas, 2001. p. 101-104.

CARDOSO, J. R. B. Resolução de problemas convencionais e não convencionais: uma análise das estratégias utilizadas por estudantes com prognóstico e diagnóstico de Discalculia. $141 \mathrm{f}$. Dissertação (Mestrado em Educação em Ciências e Matemática) - Programa de Pós-Graduação em Educação em Ciências e Matemática, PUCRS, Porto Alegre, 2019.

CARRAHER, T. N.; CARRAHER, D. W.; SCHLIEMANN, A. D. Na vida dez, na escola zero. São Paulo: Cortez, 1988.

CIASCA, S. M. Distúrbios de aprendizagem: proposta de avaliação interdisciplinar. São Paulo: Casa do Psicólogo Livraria e Editora Ltda., 2003.

CID-10. Classificação de Transtornos Mentais e de Comportamento da CID-10: Descrições clínicas e diretrizes diagnósticas. Organização Mundial de Saúde. Porto Alegre: Artes Médicas, 1993.

COHN, R. Developmental dyscalculia. Pediatric Clinics of N. America, 1968.

CONSENZA, R. N.; GUERRA, L. B. Neurociência e Educação: como o cérebro aprende. São Paulo: Artes Médicas, 2011.

CURY, H. N. Análise de erros: o que podemos aprender com as respostas dos alunos. Belo Horizonte: Autêntica, 2007.

DANTE, L. R. Didática da resolução de problemas de matemática. São Paulo: Ática, 1991.

D’AMORE, B. Epistemologia, Didática da Matemática e Práticas de Ensino. BOLEMA, Boletim de Educação Matemática, v. 20, n. 28, p. 1179-205, 2007.

DINIZ, M. I. Os problemas convencionais nos livros didáticos. In: SMOLE, K. S; DINIZ, M. I. (org.). Ler, escrever e resolver problemas: habilidades básicas para aprender matemática. Porto Alegre: Artes Médicas, 2001. p. 100-101.

DSM-5. Manual diagnóstico e estatístico de transtornos mentais. American Psychiatric Association. 5. ed. Porto Alegre: Artes Médicas, 2014.

FIORENTINI, D. Erros e acertos no ensino-aprendizagem da matemática: problematizando uma tradição cultural. In: JORNADA NACIONAL DE EDUCAÇÃO MATEMÁTICA, 1; JORNADA REGIONAL DE EDUCAÇÃO MATEMÁTICA, 14. Passo Fundo. Anais [...]. Passo Fundo: Universidade de Passo Fundo, 2006.

GOLBERT, C. Novos rumos na aprendizagem da matemática. Porto Alegre: Mediação, 2002.

GROSSI, E. P. Dificuldades com dias contatos. In: SEMINÁRIO INTERNACIONAL SOBRE DIDÁTICA DA MATEMÁTICA. Gérard Vergnaud: O campo conceitual da multiplicação. São Paulo; Porto Alegre, 2001. 
IFRAH, G. Os Números: história de uma grande invenção. Tradução de Stella Maria de Freitas Senra; revisão técnica de Antônio José Lopes. 7. ed. São Paulo: Globo, 1994.

INEP. Ministério da Educação. Brasil no PISA 2015: sumário executivo. Brasília, DF: MEC, 2016.

KAMII, C. A criança e o número. Campinas, SP: Papirus, 1998.

KAMII, C.; HOUSMAN, L.B. Crianças pequenas reinventam a aritmética: implicações da teoria de Piaget. Tradução de Cristina Monteiro. 2. ed. Porto Alegre: Artes Médicas, 2002.

KOSC, L. Developmental dyscalculia. Journal of learning disabilities, v. 7, n. 3, p. 164-177, 1974.

LARA, I. C. M. O uso da estrutura multiplicativa na resolução de problemas nos anos iniciais da educação básica. Revista Vidya, v. 31, n. 2, p. 105-122, 2011.

LARA, I. C. M.; PIMENTEL, L. S. Resolução de problemas na educação infantil: uma análise do uso da estrutura multiplicativa. Revista Vidya, v. 35, n. 1, p. 109-120, 2015.

MORAES, R.; GALIAZZI, M. C. Análise textual discursiva. 2. ed. Ijuí: Unijuí, 2011.

NUNES, T.; BRYANT, P. Crianças fazendo matemática. Porto Alegre: Artes Médicas, 1997.

POLYA, G. A arte de resolver problemas: um novo aspecto do método matemático. 2. ed. Tradução e adaptação de Heitor Lisboa de Araújo. Rio de Janeiro: Interciência, 2006.

RAMOS, L. F. Conversas sobre números, ações e operações: uma proposta criativa para o ensino de Matemática nos primeiros anos. São Paulo: Ática, 2009.

RELVAS, M. P. Neurociência e transtornos de aprendizagem: as múltiplas eficiências para uma Educação Inclusiva. Rio de Janeiro: Wak Editora, 2011.

SEABRA, A. G.; MONTIEL, J. M.; CAPOVILLA, F. C. Prova de Aritmética. In: SEABRA, A. G.; DIAS, N. M.; CAPOVILLA, F. C. Avaliação neuropsicológica cognitiva: leitura, escrita e aritmética. São Paulo: Memnon Edições Científicas, 2013. p. 97-104.

STEIN, L. M. TDE - Teste de desempenho escolar: Manual para aplicação e interpretação. São Paulo: Casa do Psicólogo, 1994.

TOLEDO, M.; TOLEDO, M. Didática da Matemática: como e dois. São Paulo: FTD, 1997.

USISKIN, Z. Concepções sobre a álgebra da escola média e utilizações das variáveis. In: COXFORD, A. F.; SHULTE, A. P. As ideias da álgebra. São Paulo: Atual, 1998. p. 1-8.

VILA, A.; CALLEJO, M. L. “O que são crenças?”. In: MATEMÁTICA para aprender a pensar: o papel das crenças na resolução de problemas. Trad. Ernani Rosa. Porto Alegre: Artes Médicas, 2006. p. 80-89.

VERGNAUD, G. A Teoria dos Campos conceituais. In: BRUN, J. Didáctica das matemáticas. Tradução de Maria José Figueiredo. Lisboa: Instituto Piaget, 1996. p. 1-26.

ZORZI, J. L. As inversões de letras na escrita: o fantasma do espelhamento. Pró-Fono: Revista de Atualização Científica, v. 13, n. 2, p. 212-218, 2001. 